\title{
ON THE NUMBER OF DISJOINT 4-CYCLES IN REGULAR TOURNAMENTS ${ }^{1}$
}

\author{
FUHONG MA AND JIN YAN ${ }^{2}$ \\ School of Mathematics \\ Shandong University \\ Jinan 250100, P.R. China \\ e-mail: yanj@sdu.edu.cn
}

\begin{abstract}
In this paper, we prove that for an integer $r \geq 1$, every regular tournament $T$ of degree $3 r-1$ contains at least $\frac{21}{16} r-\frac{10}{3}$ disjoint directed 4 cycles. Our result is an improvement of Lichiardopol's theorem when taking $q=4$ [Discrete Math. 310 (2010) 2567-2570]: for given integers $q \geq 3$ and $r \geq 1$, a tournament $T$ with minimum out-degree and in-degree both at least $(q-1) r-1$ contains at least $r$ disjoint directed cycles of length $q$.
\end{abstract}

Keywords: regular tournament, $C_{4}$-free, disjoint cycles.

2010 Mathematics Subject Classification: 05C70, 05C38.

\section{REFERENCES}

[1] Y. Bai, B. Li and H. Li, Vertex-disjoint cycles in bipartite tournaments, Discrete Math. 338 (2015) 1307-1309. doi:10.1016/j.disc.2015.02.012

[2] J. Bang-Jensen, S. Bessy and S. Thomasse, Disjoint 3-cycles in tournaments: a Proof of the Bermond-Thomassen conjecture for tournaments, J. Graph Theory 75 (2014) 284-302. doi:10.1002/jgt.21740

[3] J.C. Bermond and C. Thomassen, Cycles in digraphs-a survey, J. Graph Theory 5 (1981) $1-43$. doi:10.1002/jgt.3190050102

\footnotetext{
${ }^{1}$ The author's work is supported by NNSF of China (No. 11271230, 11671232).

${ }^{2}$ Corresponding author.
} 
[4] S. Bessy, N. Lichiardopol and J.S. Sereni, Two proofs of the Bermond-Thomassen conjecture for tournaments with bounded minimum in-degree, Discrete Math. 310 (2010) $557-560$.

doi:10.1016/j.disc.2009.03.039

[5] N. Lichiardopol, A. Pór and J.S. Sereni, A step toward the Bermond-Thomassen conjecture about disjoint cycles in digraphs, SIAM J. Discrete Math. 23 (2009) 979-992.

doi: $10.1137 / 080715792$

[6] N. Lichiardopol, Vertex-disjoint cycles in regular tournaments, Discrete Math. 312 (2012) 1927-1930.

doi:10.1016/j.disc.2012.03.009

[7] N. Lichiardopol, Vertex-disjoint directed cycles of prescribed length in tournaments with given minimum out-degree and in-degree, Discrete Math. 310 (2010) 2567-2570. doi:10.1016/j.disc.2010.06.024

[8] C. Thomassen, Disjoint cycles in digraphs, Combinatorica 3 (1983) 393-396. doi:10.1007/BF02579195

Received 30 June 2016

Revised 2 January 2017

Accepted 3 January 2017 